\title{
(If) Tribal Children's Lives Matter, Measure Them!
}

\author{
ABHAY Bang \\ Director, Society for Education, Action and Research in Community Health (SEARCH) \\ Shodhgram, Chatgaon, Dhanora, Gadchiroli, Maharashtra 442605, India.search.gad@gmail.com
}

Nothing exists, until it is measured!

Niels Bohr

M any of us will be surprised to know that as many as 104 million tribal people live in India, more than the national populations of 90 percent of the countries. How do tribal children in India live, and more importantly die? How many? Why? What can be done? These questions bother every pediatrician's conscience - sometime or other.

When the Government of India's Expert Committee on Tribal Health requisitioned for an estimate of the Infant Mortality Rate (IMR) for the tribal population in India, the office of the Census and Sample Registration System was unable to provide the same. The nation had not cared to measure death rates in tribal children. The committee, for its Report (2018) [1], had to depend on indirectly estimated rates based on the Census 2011 and the National Family Health Surveys (NFHS) 1 to 4.

But the generic term 'tribal people' incorporates within it 705 different tribes, each having unique culture, lifestyle, and hence, with a different child mortality rate. The great anthropologist Verrier Elwin had long ago documented the life and culture of various major tribes in India [1]. Who would provide us their IMRs? In the present issue of Indian Pediatrics, Verma, Sharma and Saha, three researchers from the National Institute of Research in Tribal Health, provide us exactly that [2].

From the Census of India (2011) data they have estimated the IMR, Under-5 Mortality Rate (U5MR) and Life Expectancy at Birth (LEB) for scheduled tribes (ST) from seven states - Rajasthan, Gujarat, Maharashtra, Madhya Pradesh, Chhattisgarh, Jharkhand and Odisha [2]. They selected the tribes with population size large enough, indentifying 123 tribes which account for $94-97 \%$ of the total ST population in the respective states. Using the demographic methods of indirect estimation pioneered by Prof. Brass of the London School of Tropical Medicine and Hygiene, they estimated, for the first time, the IMR, U5MR and LEB for these 123 tribes [2]. They deserve kudos for this contribution.

These estimates reveal three diversities - between the total population and the ST population of India, between the seven states, and within each state, between the tribes. Since what the authors have estimated, the IMR, U5MR and LEB, are literally the estimates of life and death, they matter! What these different statistical numbers reveal are huge inequalities for the opportunity to survive - the most fundamental human right.

These researchers estimate that whereas the IMR for the total population of India, in 2006-07, was 65 per 1000 live births, it was 76 for the total ST population ( 705 tribes) in the country. But hidden within this number, 76, were huge diversities. The IMR for these 123 tribes varied from the lowest 48 for the Gamit tribe in Maharashtra to the highest 124 for the Birhore and Bharia tribes residing in Chhattisgarh, Jharkhand and Madhya Pradesh. Seventy six additional infants died in these later two tribes per 1000 live births.

Similarly, the U5MR among these 123 tribes ranged from the lowest of 57 in one tribe to the highest of 203 in another. It was lowest for the total ST population in Maharashtra (76) and highest (123) in Madhya Pradesh. The estimated LEB for these 123 tribes ranged from the lowest 51 years (Birhore) to the highest 72 years (Gamit).

Two major limitations of these first-ever estimates are, one, they are not actual measurements over a period of time but have been indirectly estimated from a crosssectional data, the Census of India. Second, they pertain to the year 2006-07, in a way, already outdated. But they make a beginning of making estimates for the individual tribes. Hopefully, the estimates based on the next national Census will arrive sooner.

The two landmark reports, of the Lancet-Lowitja Global Collaboration (2016) on the Health of the Indigenous and Tribal Populations [3] and 'Tribal Health in India - the report of the Expert Committee on Tribal Health' of the Government of India (2018) [4], have 
pointed out that globally as well as nationally, the indigenous and tribal people suffer worse health status and chances of survival compared to the general population in the countries. Regrettably, India had the second highest IMR for the tribal people in the world, next only to Pakistan. Now Verma, Sharma and Saha show that even within the tribes, there are large disparities between the states and within the states.

\section{So, what do we make of this?}

One, the policymakers need to appreciate the importance of segregated measurement for the tribal people as a whole and for each individual tribe. The expert committee on tribal health has underscored this need; and some movement in the academia can be seen after that. Will the Ministry of Health and Family Welfare, and the Ministry of Tribal Affairs show more action?

Second, the tribal development plans - the tribal subplans - and the health plans of the states should now move further and develop the tribe specific plans. Birhore tribe, whether in Chhattisgarh, Jharkhand or Madhya Pradesh, has the highest child mortality. Each tribe has different challenges, hence needs separate attention and solutions.
Third, the pediatricians and policymakers need to assert that the tribal mothers and children receive near complete coverage with the proven health care inter-ventions such as the ANC, institutional delivery, home-based neonatal care, immunization, breastfeeding and nutrition, and finally, treatment for pneumonia, diarrhea and malaria. But the coverage will improve only if measured and monitored separately for tribal children. Niels Bohr was absolutely right - (If) tribal children lives matter, measure them!

\section{REFERENCES}

1. Guha R. Savaging the Civilized: Verrier Elwin, His Tribals, and India.University of Chicago Press, 1999.

2. Verma A, Sharma RK, Saha KB. Diversity in child mortality and life expectancy at birth among major tribes in selected states of India. Indian Pediatr. 2020;58:20-24.

3. Anderson I, Robson B, Connolly M, et al. Indigenous and tribal peoples' health, the Lancet-Lowitja institute global collaboration: A population study. Lancet. 2016;388: $131-57$.

4. Ministry of Health and Family Welfare, and Ministry of Tribal Affairs, Government of India. Tribal Health in India- Report of the Expert Committee on Tribal Health. Government of India; 2018. Accessed on December, 16, 2020. Available from: https://www.nhm.gov.in $/$ hhm $_{-}$ components/tribal_report/Executive_Summary.pdf 